\title{
Undiagnosed Bicuspid Aortic Valve: A Silent Danger
}

Ngow HA $\mathrm{H}^{\mathrm{a}}$, Wan Khairina WMN ${ }^{\mathrm{b}}$

\author{
aDepartment of Internal Medicine, Kulliyah of Medicine, International Islamic University Malaysia, \\ Kuantan, Pahang, Malaysia \\ ${ }^{b}$ Department of Paediatrics, Hospital Tengku Ampuan Afzan, 25100 Kuantan, Pahang, Malaysia
}

\begin{abstract}
The normal aortic valve has three leaflets, which are almost equal in size. A bicuspid aortic valve developed as a result of abnormal aortic cusp formation during early embryogenesis. Complications such as valvular stenosis or incompetence are well known. Delayed in identifying the congenital abnormality or the complications may cause morbidity and mortality. We report two cases of congestive cardiac failure as the results of undiagnosed bicuspid aortic valve with severe aortic incompetence. Both young men in their productive age without cardiac risk factors, were unfortunate to present too late. Although one of them was lucky to survive the ordeal, most late presentation ends up gravely. Aortic valve replacement can be offered if early detection is made. Those with severe disease at diagnosis may require a heart transplant.
\end{abstract}

KEYWORDS: Bicuspid aortic valve, aortic incompetent, heart failure, aortic valve replacement, heart transplant

\section{INTRODUCTION}

Bicuspid aortic valve is a rare congenital anomaly in our population. The incidence of the condition in this country has never been reported. The population frequency of bicuspid aortic valve from a Caucasian study is about $0.9 \%$ to $2.0 \%$, with a $2: 1$ male to female ratio. ${ }^{1}$

The association of bicuspid aortic valve with aortic stenosis, aortic regurgitation and infective endocarditis has been known for almost 150 years. It has also been recognized as a cause for dissection of the aorta for the past 75 years. ${ }^{2}$ These complications are mentioned only briefly in the majority of the cardiology textbooks. ${ }^{3}$ It is the most common reason for a predisposition to severe aortic regurgitation or stenosis in middle age patients, but they are often asymptomatic until late in the disease. Failure to detect the murmur or lost to clinical follow up can lead to dire clinical consequences. Therefore, early diagnosis and vigilant surveillance are very crucial to allow appropriate intervention, so as to preserve the cardiac function. We describe two cases of severe aortic regurgitation with decompensated heart failure as a result of the undiagnosed bicuspid aortic valve. Many patients in our community with this condition either have never been diagnosed or

Corresponding Author:

Dr. Harris Ngow Abdullah, Associate Professor, Cardiologist and Physician Department Of Internal Medicine Kulliyah of Medicine International Islamic University Malaysia PO BOX 141, 25710 Kuantan

Pahang, Malaysia

Tel No: 609-513 2797 have been reassured in childhood and lost to follow up. We thus point out the importance of regular review and surveillance of these patients before more significant cardiac dysfunctions occur.

\section{CASE REPORTS \\ Case 1}

A 37 year-old indigenous man presented with severe heart failure. He had no known medical or surgical illness. He complained of fever, cough and progressive breathlessness for two days. He had epigastric discomfort and vomiting on the day of admission. He did not have chest pain, joint pain, sore throat, skin infection or recent history of chest trauma. There was no history of travelling, swimming in the river or jungle trekking. He denied regular consumption of alcohol, recreational drug and was a non-smoker. He had no high-risk behavior.

Clinical examination revealed a thin man with the blood pressure of $115 / 59 \mathrm{mmHg}$ and tachycardia. He had a low-grade fever, and the oxygen saturation on room air was $100 \%$. He was jaundice and pale. The jugular venous pulsation was elevated at $9 \mathrm{~cm}$ from the angle of Louis. His cardiac examination revealed cardiomegaly with a soft pan-systolic murmur of grade $1 / 6$ in the tricuspid area and early diastolic murmur in the aortic area. There were fine crepitations over the lung bases. He had hepatomegaly with gross pedal edema. The chest roentgenogram showed gross cardiomegaly. The electrocardiogram showed generalized low QRS complexes without evidence of ischemic changes. A trans- thoracic echocardiography revealed a globally dilated and hypo-kinetic heart with very poor left ventricular function. The estimated ejection fraction on M-mode was $15 \%$. The LVEDd was $7.3 \mathrm{~cm}$, and LVESd was $6.4 \mathrm{~cm}$. The aortic valve was bicuspid and showing severe regurgitation. The 
vena contracta of the regurgitation jet was $0.6 \mathrm{~cm}$ with the presence of aortic holo-diastolic flow reversal. A week later, a trans-esophageal echocardiography confirmed the bicuspid aortic valve with right coronary cusp prolapse. A severe aortic regurgitation jet was seen directed posterior via color Doppler. The aorta root was not dilated. No vegetations or intra-cardiac clot was seen (Figure 1 and Figure 2).

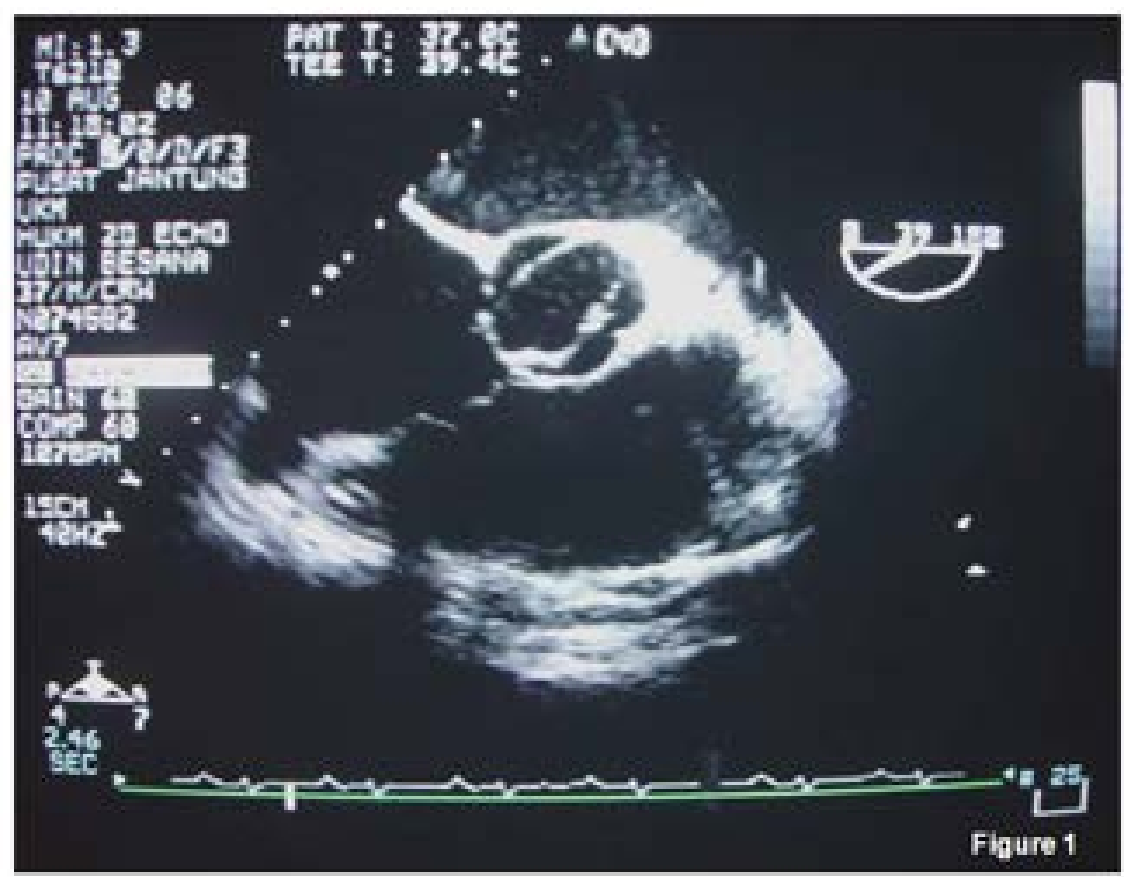

The trans-esophageal echocardiography of first case showing bicuspid aortic valve.It It was taken during systole which clearly showed the opening of only 2 leaflets with 22 commeasures

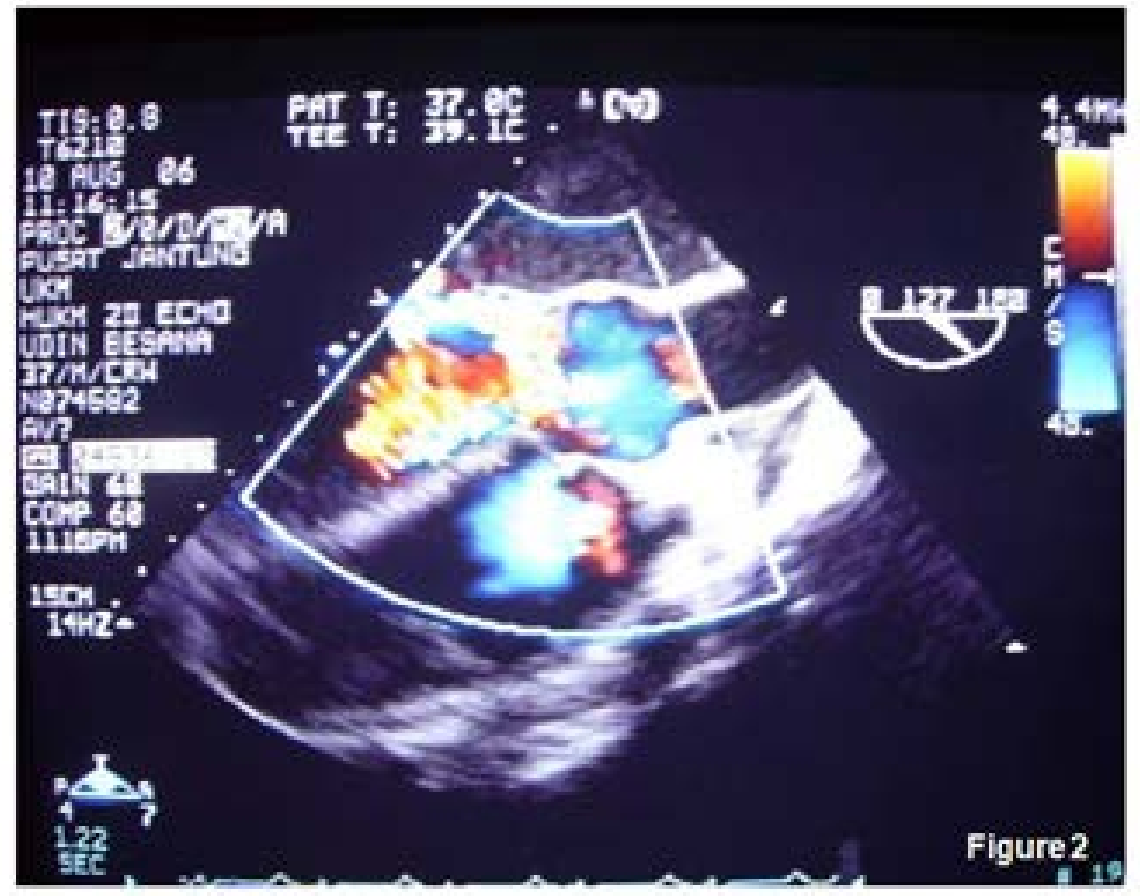

Severe posterior jet of aortic regurgitation during diastole with prolapsed of the posterior aortic valve leaflet. 
The patient was referred to our cardiothoracic surgeon for aortic valve replacement while he was stabilized on anti-failure medications. He also developed severe ischemic hepatitis as a result of the poor cardiac function and required ventilatory support for respiratory failure. He later acquired nosocomial pneumonia and despite vigorous treatment, the patient succumbed to his illness without the opportunity for aortic valve replacement.

\section{Case 2}

A 27 years-old soldier was admitted to our hospital with dyspnoea and reduced effort tolerance. He did not have fever, angina, palpitation and syncope. There was no family history of ischemic heart disease. He had no similar symptoms prior to this presentation. He was a non-smoker and teetotal. He denied any recent severe stress or prolonged travelling and was not on any traditional treatment or recreational drug.
His compulsory pre-employment medical examination and cardiac assessment were normal.

On admission, he was in respiratory distress, requiring oxygen therapy. The cardiovascular examination showed collapsing pulse, Corrigan's sign and elevated jugular venous pulsation at $6 \mathrm{~cm}$ from the sterna angle. An early diastolic murmur was heard at the left parasternal region and a grade 3/6 pan-systolic murmur in the mitral region. He was in heart failure with bi-basal fine crackles in the lungs. His chest roentgenogram showed gross cardiomegaly and pulmonary oedema. Laboratory investigations were unremarkable.

The electrocardiogram showed left ventricular hypertrophy with widespread $\mathrm{T}$ wave abnormality (Figure 3).

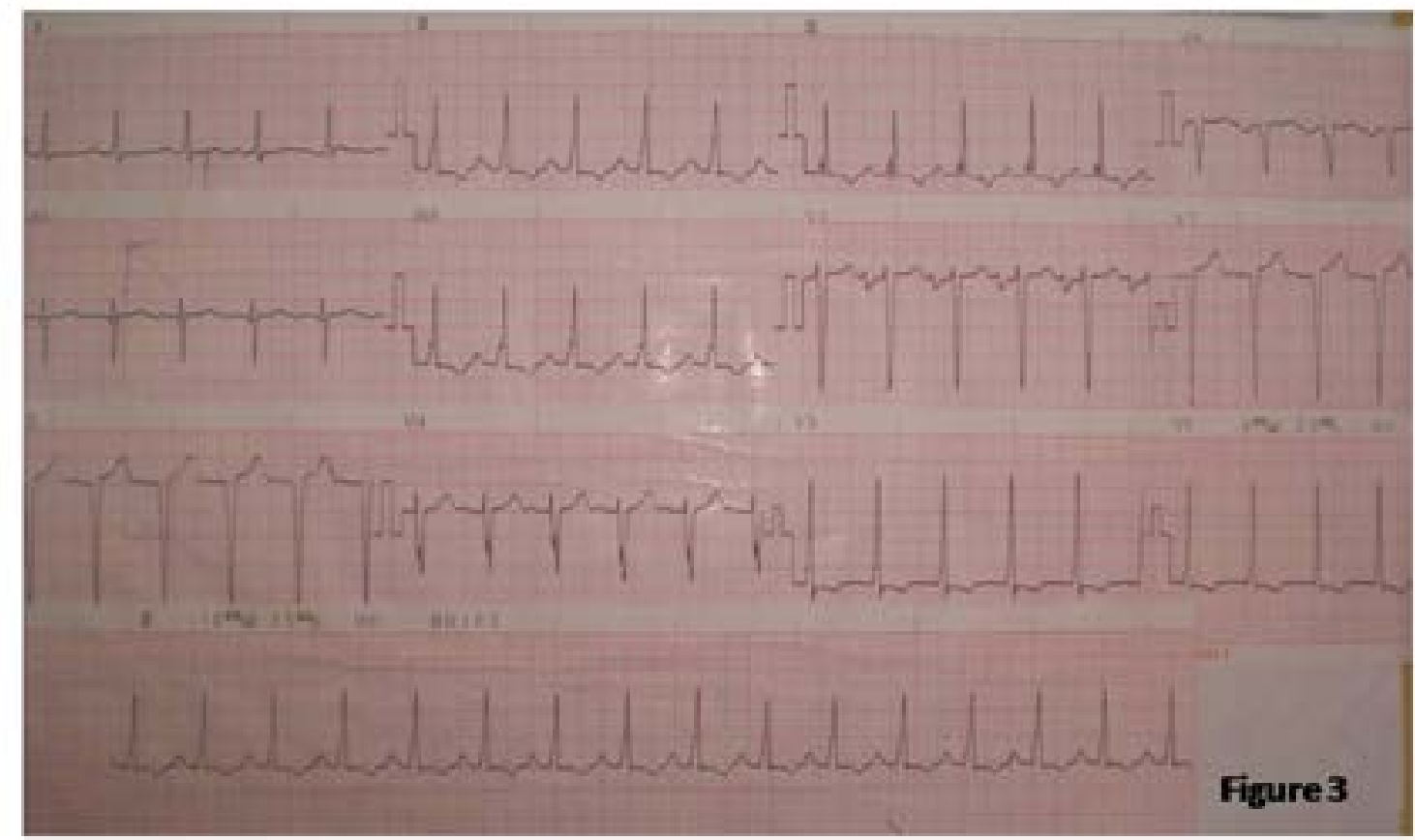

Electrocardiogram of case 2 shows sinus tachycardia and left ventricular hypertrophy with diffuse $\mathrm{T}$ wave abnormality

The 2-Dimensional echocardiogram showed grossly dilated heart with global hypokinesia. There were also moderate mitral regurgitation and severe aortic regurgitation. The estimated ejection fraction on M-mode was $20 \%$. The LVEDd was $6.8 \mathrm{~cm} \mathrm{~cm}$ and LVESd was $5.6 \mathrm{~cm}$. The aortic regurgitation vena contracta was $0.7 \mathrm{~cm}$ and there was a presence of aortic holo-diastolic reversal. The mitral regurgitation vena contracta was $0.4 \mathrm{~cm}$ with regurgitation volume of $48 \mathrm{ml}$ and regurgitation fraction of $42 \%$. The trans-esophageal echocardiography later revealed bicuspid aortic valve with severe aortic regurgitation. He responded to intravenous diuretics and stabilized with anti-failure medications. He was subsequently referred for aortic valve replacement and enrolled into the heart transplant program in a tertiary hospital. Four months after the aortic valve replacement, he remained well and had not been admitted for heart failure.

\section{DISCUSSION}

We report two cases of under-diagnosed bicuspid aortic valve, which led to grave and fatal consequences. This congenital anomaly occurs when the aortic valve does not develop normally. The reported incidence of the condition in published literature is about $0.9 \%$ to $2.0 \%$ with a male preponderance. ${ }^{1}$ The incidence of aortic regurgitation arising from bicuspid aortic valve is estimated at $1.5 \%$ to $3.0 \% .4$ The valvular incompetence may develop due to prolapsed of the greater cusp or a direct consequence of aortic root dilatation, aortic coarctation or endocarditis. ${ }^{5}$ In this condition, it is suggested that if no significant stenosis or only trivial insufficiency is present, significant haemodynamic changes will not develop before the age of 70.6 However, based on published reports, the majority of patients will have died or developed serious symptoms requiring surgical intervention by this age. 
Clinical diagnosis of bicuspid aortic valve can be made on the presence of aortic ejection click, regardless of whether ejection systolic murmur is present or not. This abnormality was insufficiently diagnosed before the widespread use of two-dimensional echocardiography. The reliability of the diagnosiscan be significantly improved with cross-sectional and Doppler echocardiography with a specificity of $98 \%$, a sensitivity of $78 \%$ and a positive predictive value of $93 \% .^{7}$ Diagnosis is made based on visualization of 2 cusps and 2 commissures during short axis view. However, the usually bicuspid nature of valve may be obscured by severe calcification and fibrosis. A false-positive result may occur when one coaptation of the line of the tri-leaflet valve is unclear or as a result of a prominent raphe during echocardiography study. A medical examination and even an echocardiography may miss the condition before complications develop. Since there is no specific age prevalence at which majority acquires valvular complications, these patients thus require regular examination and echocardiography. As illustrated in case 1 , the patient was diagnosed late as he was staying in a remote area where echocardiography examination was poorly accessible. In addition, the treating physician should maintain a high index of suspicion of bicuspid aortic valve, especially when aortic valve disease was detected in the young. This has led to the late diagnosis and dire consequences in both patients as illustrated in our report.

The etiology of aortic incompetence in bicuspid aortic valve is rather complex. It may occur in isolation, usually as a result of prolapsed of the larger of unequally sized cusps or in association with aortic root dilatation or infective endocarditis. ${ }^{8}$ In the first case, we believe that the acute presentation was a result of a recent prolapsed posterior leaflet of a bicuspid aortic valve. However, judging from the already dilated left ventricular chamber size, the timing of the prolapsed valve might have been earlier. As an isolated phenomenon, a bicuspid aortic valve is a relatively uncommon cause of severe aortic regurgitation. Nevertheless, if the incidence of rheumatic heart disease is added, the incidence of aortic incompetent in bicuspid aortic valve increases significantly from $3 \%$ to $40 \% .{ }^{1}$ However, in both of our patients, there was no evidence of rheumatic or infective endocarditis. Because of the declining incidence of rheumatic heart disease, it appears that bicuspid aortic valve may be associated directly or indirectly with the majority of cases of severe aortic incompetent. ${ }^{9}$ Due to its association with infective endocarditis and coartation of aorta, most patients with aortic regurgitation die or have aortic valve replacement at an earlier age than those with pure aortic stenosis.

From our review, the incidence of bicuspid aortic valve's complication may be over-estimated. In childhood and early adult life, critical stenosis and infective endocarditis are the commonest complication requiring surgical intervention. From early adulthood till middle age, aortic regurgitation is Volume 11 Number 2, Dec 2012 the major morbidity. As illustrated in our cases, late diagnosis can even cause mortality.

Therefore, in our opinion, all patients with bicuspid aortic valve should undergo early intervention when complications were detected. Other risk factors such as smoking, hypertension and hypercholesterolemia should be treated and meticulously controlled. Screening of the first-degree relative may be appropriate in view of familial clustering of this condition. ${ }^{10}$ It was indeed unfortunate for one of our patients who succumbed to the complication of unrecognized icuspid aortic valve and severe aortic incompetence. For the patient who escapes death with severely dilated heart, aortic valve replacement may be a temporary measure prior to heart transplantation.

\section{REFERENCE}

1. Saga S, Bastiaenen R, Hayward M, McEwan JR. An undiagnosed bicuspid aortic valve can result in severe left ventricular failure. BMJ 2007;334:420-2.

2. Peacock TB. Valvular disease of the heart. London: Churchill, 1865:2-33.

3. Perloff JK. Congenital heart disease in adult. In: Braunwald E, 8th ed. Heart disease, a textbook of cardiovascular medicine, 8th ed. Philadelphia: WB Saunders, 2006; 969.

4. Roberts WC. The congenitally bicuspid aortic valve: A study of 85 autopsy cases. Am J Cardiol 1970;26:72-83.

5. Fenoglio JJ, McAllister HA, DeCastro CM,Davio JE, Cheitlin MD. Congenital bicuspic aortic valve after age 20. Am J Cardiol 1997;39:164-9.

6. Zeppilli P, Bianco M, Bria S, Palmieri V. Bicuspid aortic valve: an innocent finding or a potentially life-threatening anomaly whose complications may be elicited by sports activity? J Cardiovasc Med 2006;7: 282-7.

7. Weyman AE, Griffin BP. Left ventricular outflow tract: the aortic valve, aorta and subvalvular outflow tract. In: Weyman AE, ed. Principles and practice of echocardiography, 2nd Ed. Philadelphia: Lea and Febiger, 1994:505-8.

8. Ward C. Clinical significance of the bicuspia aortic valve. Heart 2000;83:81-.5

9. Grant RT, Wood JE, Jones TD. Heart valve ir regularities in relation to sub-acute bacterial endocarditis. Heart 1928;14:247-55.

10. Huntington K, Hunter AGW, Chan KL. A prospective study to assess the frequency of familial clustering of congenital bicuspid aortic valve. J Am Coll Cardiol 1997;30: 1809-12. 